\title{
ECoG spectrum changes at different xenon-isoflurane anaesthesia depths
}

\author{
Bogdan Pavel ${ }^{1,2}$, Camelia Alexandra Acatrinei ${ }^{1}$, Maria Corbu ${ }^{1}$, Carmen Mihaela Denise Zahiu ${ }^{1}$, Adrian \\ Eugen Rosca ${ }^{1}$, Leon Zagrean ${ }^{1}$, Ana-Maria Zagrean ${ }^{1}$ \\ 1 "Carol Davila" University of Medicine and Pharmacy, Division of Physiology and Fundamental Neurosciences, Bucharest, Romania \\ ${ }^{2}$ Clinical Emergency Hospital of Plastic Surgery and Burns, Bucharest, Romania
}

\begin{abstract}
Background and aims. The purpose of this study is to assess the frontal and parietal ECoG spectrum (gamma range) changes during isoflurane and combined xenon-isoflurane anaesthesia in rats.

Methods. Experiments were carried out on four adult male Sprague-Dawley rats (250-300 g). The anaesthesia was induced with isoflurane and maintained with isoflurane and a xenon-isoflurane mixture. The rats were maintained at two different anaesthetic depths: light (isoflurane anaesthesia) and deep (isoflurane and xenon-isoflurane anaesthesia). The frontal and the parietal cortical activity was assessed by computing the median frequency, spectral edge frequency and functional connectivity between these two areas during light and deep anaesthesia.

Results. We noticed a decrease in cortical connectivity under deep isoflurane anaesthesia and an increase in connectivity under deep xenon-isoflurane anaesthesia. Moreover, during xenon-isoflurane anaesthesia, a trend of regularity of electro-cortical activity was present compared with isoflurane anaesthesia.

Conclusions. Xenon-isoflurane deep anaesthesia demonstrated a series of specific ECoG features regarding frontoparietal functional connectivity (gamma range connectivity increase) and regularity of the electrocortical activity compared with isoflurane anaesthesia.
\end{abstract}

Keywords: ECoG spectrum, xenon, isoflurane, anaesthesia

Rom J Anaesth Int Care 2017; 24: 41-46

\section{Introduction}

During anaesthesia, EEG spectrum modifications appear, consistent with the anaesthetic depth $[1,2]$. These changes vary from low frequencies high amplitude EEG waves to burst suppression patterns and depend, among other things, on the type of anaesthetic used and the age of the patient [3-5]. On the other hand, loss of consciousness (LOC) is associated with

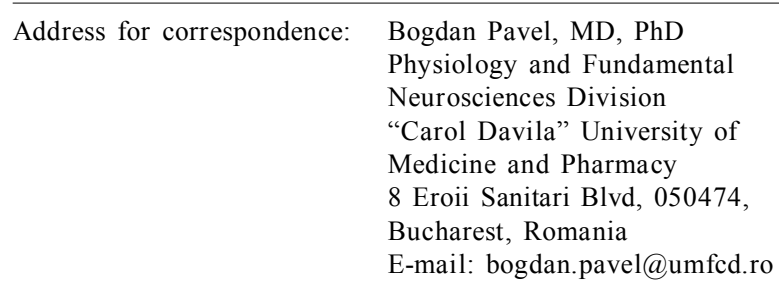

a decrease of activity and connectivity in the gamma domain $[6,7]$. Xenon is a gas whose anaesthetic properties have been known since the 1940s, but its use in anaesthetic practice only began in the last 20 years $[8,9]$. Few studies have investigated the xenoninduced EEG modifications, and most depth of anaesthesia monitors are not validated for xenon [10, $11]$.

In rats, the anaesthetic xenon concentration is hyperbaric at 1.61 atmospheres, so, to study the xenon effects, a xenon-isoflurane combined anaesthesia is required [12]. We consider that the ECoG modifications during xenon-isoflurane combined anaesthesia are due to the adding of xenon to the isoflurane because xenonisoflurane combined anaesthesia induces a particular pattern, not noticed in pure isoflurane anaesthesia, but it cannot be considered a pure substitute for xenon anaesthesia. 
In this study, we have investigated the $\mathrm{ECoG}$ activity modifications in rats, under isoflurane and xenon-isoflurane combined anaesthesia, in the gamma range. As far as we know, this is the first study performed in Romania that investigates the anaesthetic effects of xenon.

\section{Materials and methods}

Experiments were carried out on adult male Sprague-Dawley rats $(\mathrm{n}=4)$, weighing between 250 and $300 \mathrm{~g}$, with water and food ad libitum. We conducted this study with the approval of the local committee for animal research of "Carol Davila" University of Medicine and Pharmacy (Bucharest, Romania) and in agreement with the European Communities Council Directive 86/609/EEC for the protection of animals used for scientific purposes.

Chronic electrode implantation technique

Firstly, the head of the rat was shaved. A three centimeters long incision was performed centrally. Skin and subcutaneous tissue were removed from the skull. Afterwards, a few drops of iron chloride were applied for one minute; the bone surface was cleaned using physiological saline solution and disinfected using povidone-iodine.

Secondly, the rat was mounted in the stereotaxic ear bars. The Bregma and Lambda points were marked, and holes were drilled into the bone: $4 \mathrm{~mm}$ anterior and $3 \mathrm{~mm}$ lateral above the frontal cortex; $3 \mathrm{~mm}$ lateral and $3 \mathrm{~mm}$ posterior above the parietal cortex corresponding to the somatosensory region. For the reference electrode, a hole was drilled $10 \mathrm{~mm}$ anterior and $1 \mathrm{~mm}$ lateral left to the Bregma. Paxinos and Watson's "The Rat Brain in Stereotaxic Coordinates" was used as reference for the coordinates [13].

After drilling, a plastic head cap with Ni-Chrome electrodes was set, with the electrodes in direct contact with the dura mater. The entire electrode assembly was insulated and bonded to the skull with dental UnifastTrad Cement. Recordings were performed 7 days after electrode implantation.

\section{Isoflurane and xenon-isoflurane anaesthesia protocol}

Anaesthesia was induced using $4 \%$ isoflurane, using an anaesthesia machine (Ugo Basile, Italy) in an induction chamber, at a gas flow of 4 liters per minute, for three minutes. After induction, the rats were orotracheally intubated and connected to a mechanical ventilator for rats (SAR-830/AP ventilator, CWE Inc.). Assessing the cortical electrical activity, we have noticed a deep burst suppression pattern, following the $4 \%$ isoflurane induction. $1.1 \%$ isoflurane anaesthesia was administered, and we waited until the cortical electrical activity developed a slow wave pattern (this period varied among rats, with an average of 10 minutes). Afterwards, we waited another 10 minutes at the concentration of $1.1 \%$ and then lowered it to $0.9 \%$, allowing 5 minutes for balancing and then recording another 5 minutes. At the end, the isoflurane concentration was decreased to $0.5 \%$ and $50 \%$ xenon (Linde Gas, > 99.999\%) was administered for 4 minutes in order to get a deep slow wave anaesthesia (we noticed that while adding xenon, the ECoG modifications were much quicker to appear than expected, as Xenon is a gas).

To estimate the anaesthetic depth we chose, by convention, the frontal lobe electrical activity. Light anaesthesia was considered at a MEF (in the frontal lobe) value greater than $5.5 \mathrm{~Hz}$, while deep anaesthesia was considered at a MEF value lower than $4.5 \mathrm{~Hz}$.

\section{Data acquisition and processing}

Signal was acquired using the MP150 Data Acquisition System - Biopac, at an acquisition frequency of $1000 \mathrm{~Hz}$ and signal amplification of 1000 . To record gamma frequencies, the $35 \mathrm{~Hz}$ filter was removed. The two 1-minute tracings - corresponding to the light and deep anaesthesia - were selected. 4 epochs of 3 seconds corresponding to these recordings were analyzed, and the resulted values were subsequently averaged.

\section{Data analysis}

The recordings were saved in the .acq and .mat format to be processed using the AcqKnowledge 4.2 and the MATLAB programs.

Using the MATLAB function mscohere, the frontoparietal functional connectivity was estimated. The cortical activity during different anaesthetic depths was assessed using median frequency (MEF), and spectral edge frequency (SEF) functions from AcqKnowledge 4.2. The data obtained was processed in the SPSS 12.0 software, using t student test. The Levene test was used to assess the mean variance. Results are presented as mean \pm standard deviation. A p-value $<0.05$ was considered statistically significant.

\section{Results}

The following results were obtained from the ECoG recordings analysis during isoflurane light and deep anaesthesia and xenon-isoflurane deep anaesthesia. Frontal ECoG tracings are presented during deep (Fig. 1) and light (Fig. 2) isoflurane anaesthesia. Xenonisoflurane ECoG features are shown in Figure 3.

During light isoflurane anaesthesia, frontoparietal connectivity in the gamma range $(35-45 \mathrm{~Hz})$ was 0.47 \pm 0.23 , compared with deep isoflurane anaesthesia, 


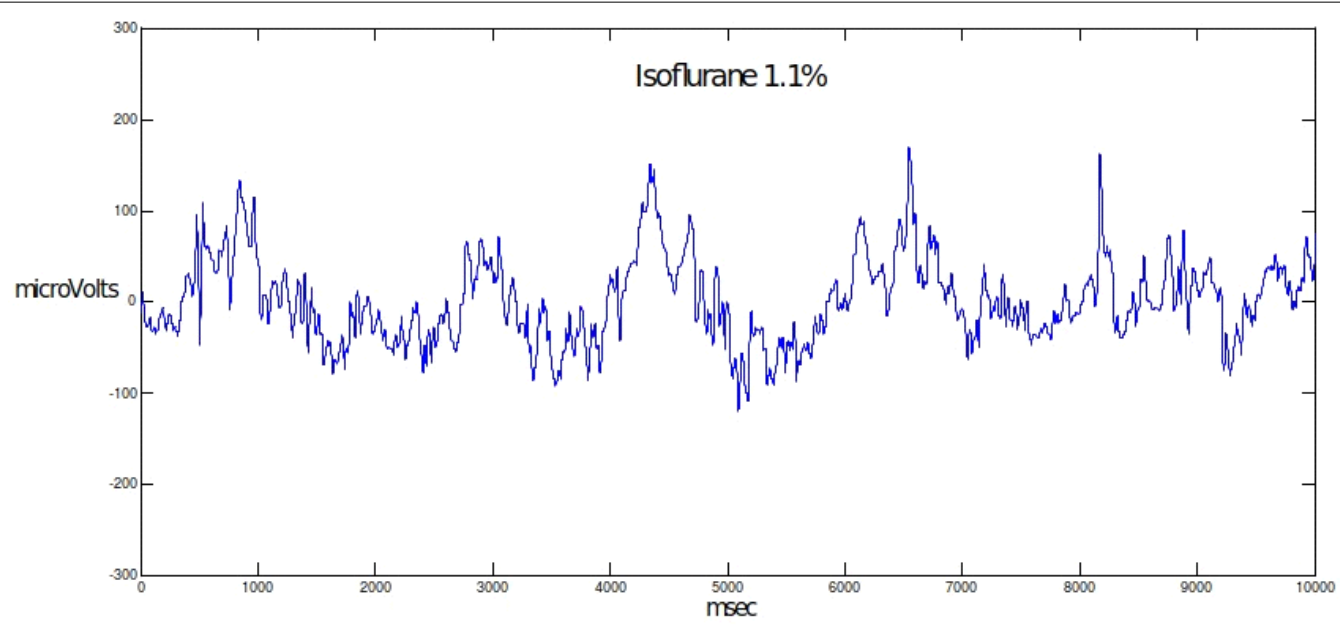

Fig. 1. ECoG frontal recording under $1.1 \%$ isoflurane anaesthesia

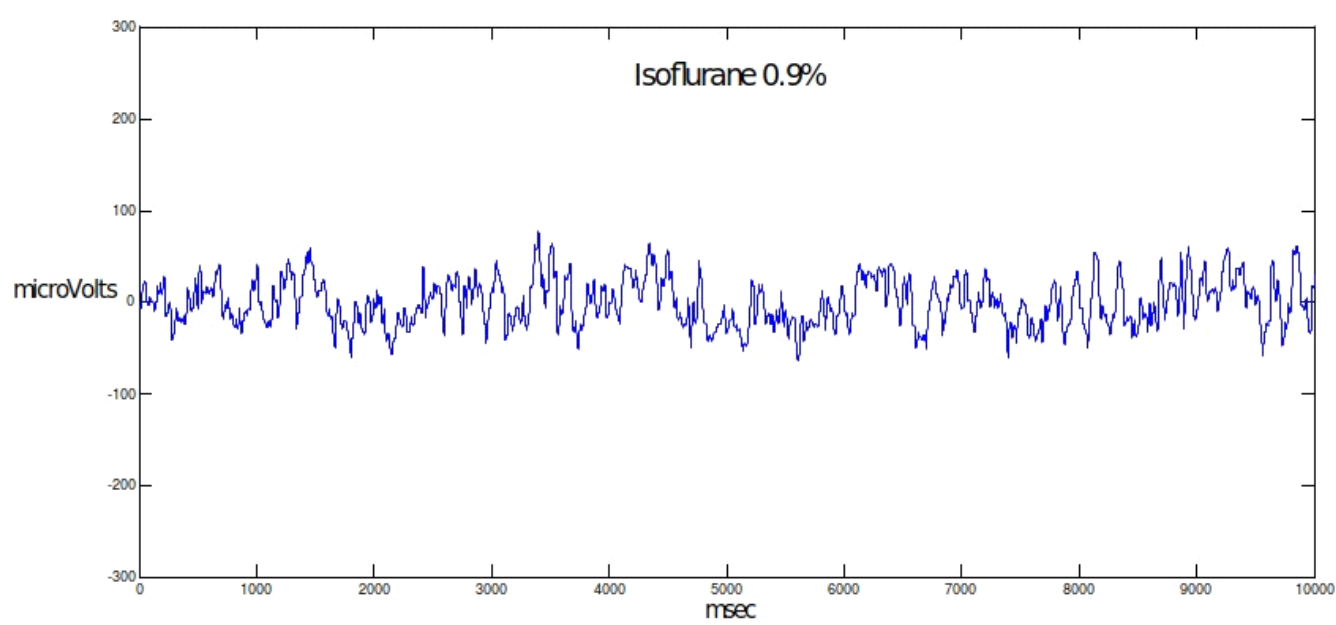

Fig. 2. ECoG frontal recording under $0.9 \%$ isoflurane anaesthesia

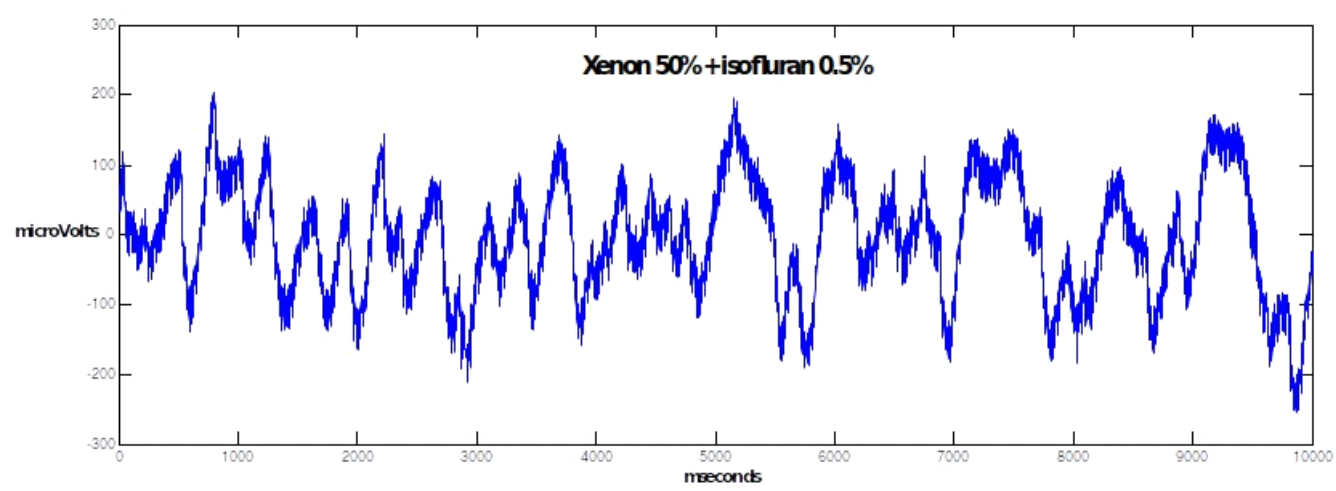

Fig. 3. ECoG frontal recording under xenon-isoflurane anaesthesia

for which frontoparietal connectivity was $0.14 \pm 0.09$ $(p<0.05)$ (Fig. 4 and Fig. 5). Under xenon-isoflurane deep anaesthesia, frontoparietal connectivity increased to $0.3 \pm 0.04(p<0.05)$ (Fig. 6 and Fig. 7). Table 1 presents the median frequency and spectral edge frequency values in frontal and parietal areas during light and deep isoflurane anaesthesia, as well as during xenon-isoflurane anaesthesia. These data revealed that, during xenon-isoflurane anaesthesia, cortical electrical activity in frontal and parietal areas becomes comparable ( $p$-value was $>0.05$ when MEF and SEF between frontal and parietal lobes were compared). 


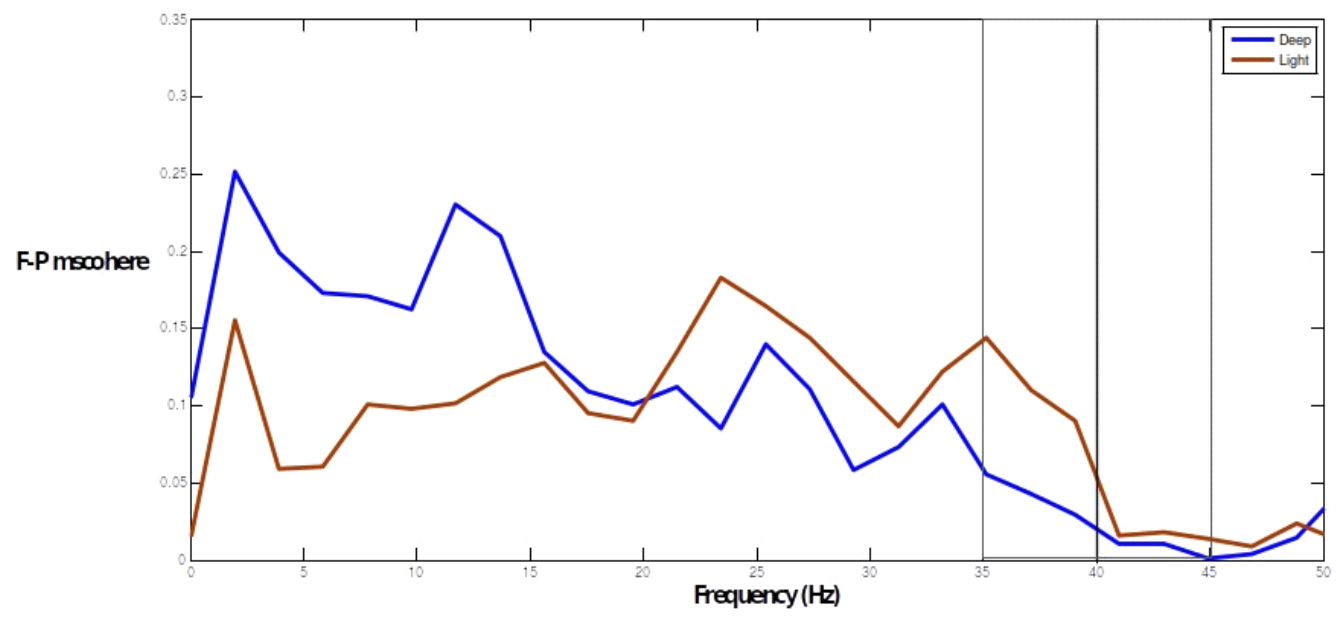

Fig. 4. Frontoparietal connectivity during deep (blue) vs. light isoflurane anaesthesia (red). The vertical markings define gamma frequency spectrum $(35-45 \mathrm{~Hz})$

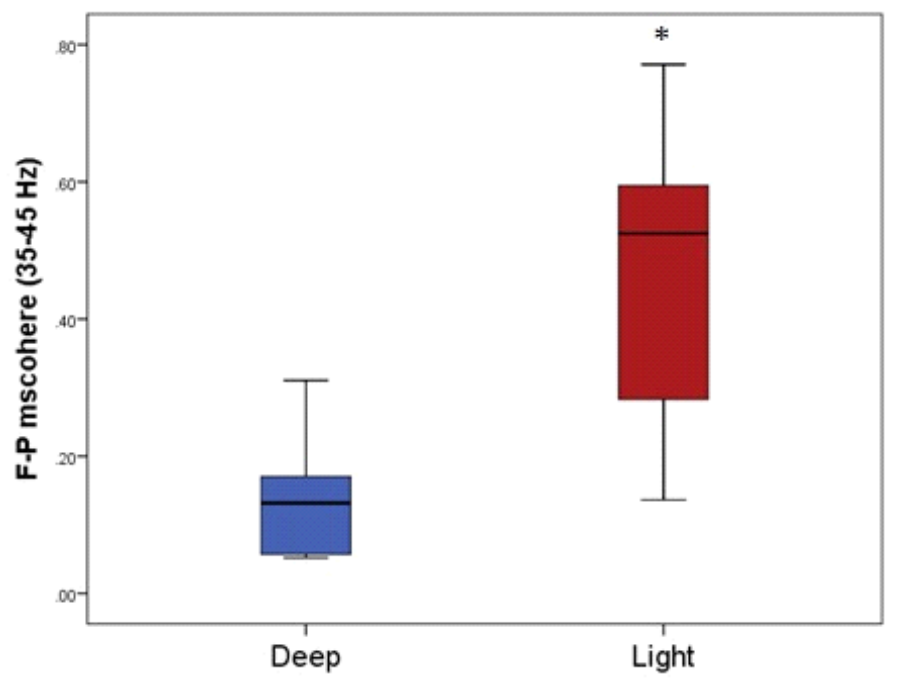

Fig. 5. Mean values of frontoparietal connectivity during deep (blue) vs. light isoflurane anaesthesia (red). Error bars represent standard deviation. * indicates $\mathrm{p}<0.05$

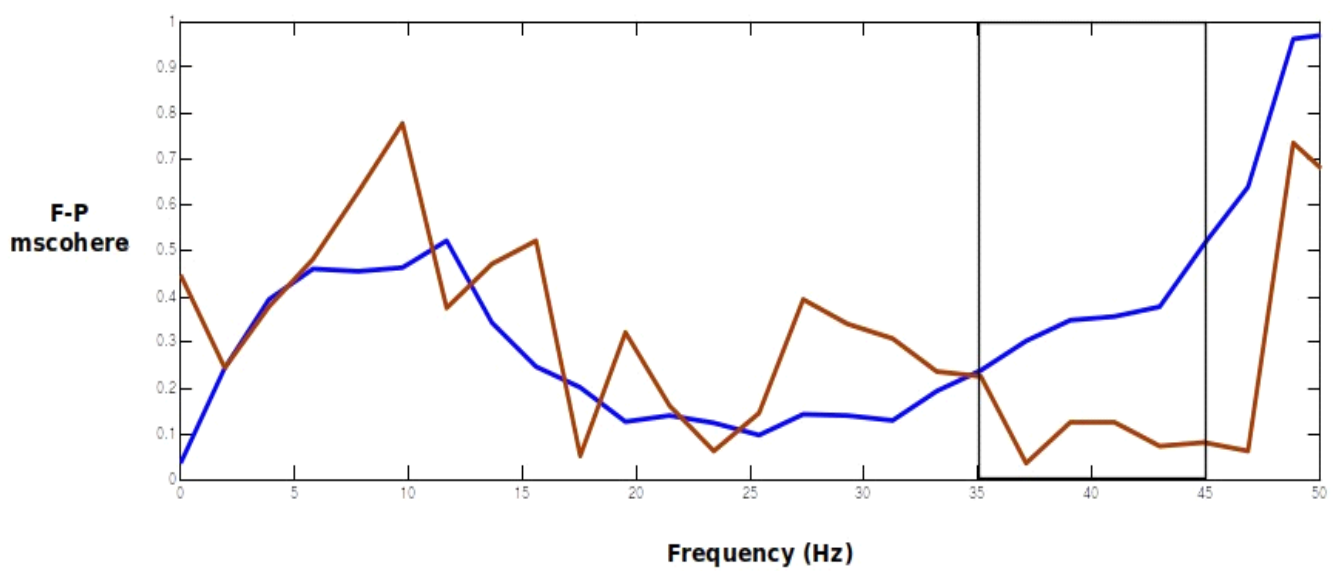

Fig. 6. Frontoparietal connectivity during deep xenon-isoflurane (blue) vs. light isoflurane anaesthesia (red). The vertical markings define gamma frequency spectrum $(35-45 \mathrm{~Hz})$ 


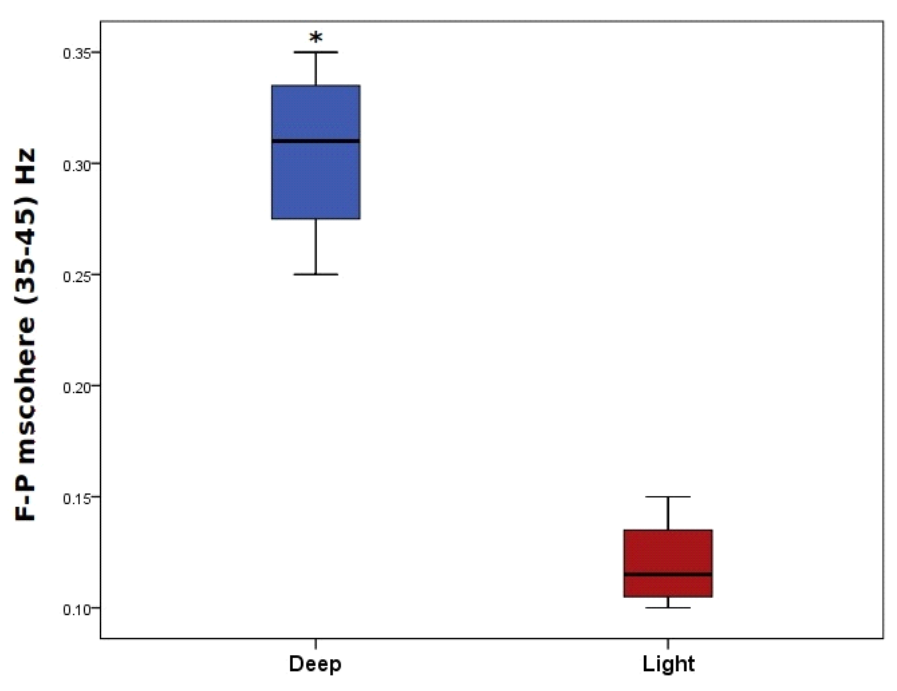

Fig. 7. Mean values of frontoparietal connectivity during deep xenon-isoflurane anaesthesia (blue) vs. light isoflurane anaesthesia (red). Error bars represent standard deviation. $*$ indicates $\mathrm{p}<0.05$

Table 1. Median frequency and spectral edge frequency values during light and deep isoflurane anaesthesia and deep xenon-isoflurane anaesthesia

\begin{tabular}{llccc} 
& & Frontal & Parietal & Statistical significance \\
\hline \multirow{2}{*}{ MEF (Hz) } & Light anaesthesia & $5.7 \pm 0.8$ & $7.4 \pm 1.73$ & $\mathrm{p}<0.05$ \\
& Deep anaesthesia & $4.18 \pm 0.77$ & $3 \pm 0.58$ & $\mathrm{p}<0.05$ \\
& Deep anaesthesia Xenon + Isoflurane & $2.55 \pm 0.26$ & $2.8 \pm 0.31$ & $\mathrm{p}=0.31$ \\
\multirow{3}{*}{ SEF (Hz) } & $28.0 \pm 1.97$ & $36.5 \pm 1.24$ & $\mathrm{p}<0.05$ \\
& Light anaesthesia & $19.8 \pm 1.68$ & $21.98 \pm 2.38$ & $\mathrm{p}<0.05$ \\
& Deep anaesthesia & $6.88 \pm 0.8$ & $7.06 \pm 0.86$ & $\mathrm{p}=0.35$ \\
& Deep anaesthesia Xenon + Isoflurane & &
\end{tabular}

Values are presented as a mean \pm standard deviation

\section{Discussion}

Our results show a decrease in frontoparietal connectivity during deep anaesthesia with isoflurane $(1.1 \%$ concentration) in the gamma range $(35-45 \mathrm{~Hz})$. These results are consistent with those of previous studies, showing that anaesthesia deepens as cortical connectivity lowers [7]. However, these studies were performed on different types of anaesthetics, both volatile (sevoflurane) and intravenous (propofol) [14]. Even in the case of ketamine that increases the gamma activity during anaesthesia induction, the frontoparietal cortical connectivity lowers [14].

It is also known that the loss of consciousness is associated with a sudden decrease in gamma domain activity [7]. To our knowledge, no studies investigated the cortical connectivity in the gamma domain during xenon-isoflurane combined anaesthesia. Surprisingly, our results show that during deep xenon-isoflurane anaesthesia, cortical frontoparietal connectivity increases in the gamma domain.

The mechanism of action of xenon is not entirely understood. Although it is known as a NMDA receptor antagonist, like ketamine, it has different effects on the cerebral electrical activity and the cerebral metabolism $[15,16]$. Ketamine, unlike other anaesthetics, does not reduce the cortical electrical activity during anaesthesia, but it does reduce the cortical connectivity in the gamma domain $[14,17]$, while xenon reduces the cortical electrical activity, but increases the cortical connectivity in the gamma domain during deep anaesthesia. Regarding the action on cerebral metabolism, xenon, unlike ketamine, produces a global decrease in metabolic activity $[18,19]$. Therefore, these changes observed during xenon anaesthesia do not appear to be linked to the action on the NMDA receptor.

During isoflurane anaesthesia, the cortical electrical activity in the frontal lobe is different from that in the parietal lobe, as shown by the median frequency and the spectral edge frequency values, during both superficial and deep anaesthesia. During deep xenonisoflurane anaesthesia, cortical electrical activity seems to have levelled out, since electrical activity in the frontal cortex is similar with the one in the parietal cortex, in terms of anaesthetic depth. This aspect can 
be easily explained in the case of combined xenonisoflurane anaesthesia, as it was noticed that during xenon anaesthesia a global decrease in cerebral metabolic activity appears in humans. In the case of isoflurane anaesthesia, a study performed on 5 patients - which assessed the relationship between anaesthetic concentration and cerebral metabolism, using PET showed a uniform decrease in metabolic cerebral activity, compared with halothane or propofol anaesthesia [20-22].

The limitations of our study are the small number of rats used and the limited number of electrodes used to assess cortical electrical activity.

\section{Conclusions}

Frontoparietal cortical connectivity (in the gamma range 35-45 Hz) increased during light anaesthesia, compared with deep anaesthesia. During xenonisoflurane deep anaesthesia, an unexpected increase in gamma connectivity was observed.

A uniformity of cortical electrical activity in frontal and parietal areas was observed during xenonisoflurane combined anaesthesia, compared with pure isoflurane anaesthesia.

\section{Conflict of interest}

Nothing to declare

\section{References}

1. Sloan TB. Anesthetic effects on electrophysiologic recordings. J Clin Neurophysiol 1998; 15: 217-226

2. Hagihira S. Changes in the electroencephalogram during anaesthesia and their physiological basis. Br J Anaesth 2015; 115 Suppl 1: i27-i31. DOI: 10.1093/bja/aev212

3. Hering W, Geisslinger G, Kamp HD, Dinkel M, Tschaikowsky $\mathrm{K}$, Rügheimer $\mathrm{E}$, et al. Changes in the EEG power spectrum after midazolam anaesthesia combined with racemic or S- $(+)$ ketamine. Acta Anaesthesiol Scand 1994; 38: 719-723. DOI: 10.1111/j.1399-6576.1994.tb03984.x

4. Rylova AV, Sazonova OB, Lubnin AIu, Masherov EL. Changes in brain bioelectrical activity during xenon anesthesia in neurosurgical patients. Anesteziol Reanimatol 2010; (2): 31-33

5. Schultz B, Schultz A, Grouven U, Zander I, Pichlmayr I. Changes with age in EEG during anesthesia. Anaesthesist 1995; 44: 467472

6. John ER, Prichep LS. The anesthetic cascade: A theory of how anesthesia suppresses consciousness. Anesthesiology 2005; 102: 447-471

7. Imas OA, Ropella KM, Ward BD, Wood JD, Hudetz AG. Volatile anesthetics disrupt frontal-posterior recurrent information transfer at gamma frequencies in rat. Neurosci Lett 2005; 387: 145-150. DOI: $10.1016 /$ j.neulet.2005.06.018

8. Lawrence JH, Loomis WF, Tobias CA, Turpin FH. Preliminary observations on the narcotic effect of xenon with a review of values for solubilities of gases in water and oils. J Physiol 1946; 105: 197-204

9. Lachmann B, Armbruster S, Schairer W, Landstra M, Trouwborst A, Van Daal GJ, et al. Safety and efficacy of xenon in routine use as an inhalational anaesthetic. Lancet 1990; 335: 14131415. DOI: $10.1016 / 0140-6736(90) 91444-F$

10. Sal'nikov PS, Burov NE. Electrophysiological features of xenon anesthesia. Anesteziol Reanimatol 2003; (1): 12-17

11. Goto T, Nakata Y, Saito H, Ishiguro Y, Niimi Y, Suwa K, et al. Bispectral analysis of the electroencephalogram does not predict responsiveness to verbal command in patients emerging from xenon anaesthesia. Br J Anaesth 2000; 85: 359-363. DOI: $10.1093 / \mathrm{bja} / 85.3 .359$

12. Koblin DD, Fang Z, Eger EI $2^{\text {nd }}$, Laster MJ, Gong D, Ionescu P, et al. Minimum alveolar concentrations of noble gases, nitrogen, and sulfur hexafluoride in rats: helium and neon as nonimmobilizers (nonanesthetics). Anesth Analg 1998; 87: 419424. DOI: $10.1213 / 00000539-199808000-00035$

13. Paxinos G, Watson C. The Rat Brain in Stereotaxic Coordinates. $6^{\text {th }}$ edition. London: Elsevier Academic Press; 2007

14. Lee U, Ku S, Noh G, Baek S, Choi B, Mashour GA. Disruption of frontal-parietal communication by ketamine, propofol, and sevoflurane. Anesthesiology 2013; 118: 1264-1275. DOI: 10.1097/ALN.0b013e31829103f5

15. Yamakura T, Harris RA. Effects of gaseous anesthetics nitrous oxide and xenon on ligand-gated ion channels. Comparison with isoflurane and ethanol. Anesthesiology 2000; 93: 10951101.

16. Franks NP, Dickinson R, de Sousa SL, Hall AC, Lieb WR. How does xenon produce anaesthesia? Nature 1998; 396: 324. DOI: $10.1038 / 24525$

17. Hans P, Dewandre PY, Brichant JF, Bonhomme V. Comparative effects of ketamine on Bispectral Index and spectral entropy of the electroencephalogram under sevoflurane anaesthesia. $\mathrm{Br} \mathrm{J}$ Anaesth 2005; 94: 336-340. DOI: 10.1093/bja/aei047

18. Rex S, Schaefer W, Meyer PH, Rossaint R, Boy C, Setani K, et al. Positron emission tomography study of regional cerebral metabolism during general anesthesia with xenon in humans. Anesthesiology 2006; 105: 936-943

19. Långsjö JW, Salmi E, Kaisti KK, Aalto S, Hinkka S, Aantaa R, et al. Effects of subanesthetic ketamine on regional cerebral glucose metabolism in humans. Anesthesiology 2004; 100: 1065-1071

20. Alkire MT, Haier RJ, Shah NK, Anderson CT. Positron emission tomography study of regional cerebral metabolism in humans during isoflurane anesthesia. Anesthesiology 1997; 86: 549557

21. Alkire MT, Pomfrett CJ, Haier RJ, Gianzero MV, Chan CM, Jacobsen BP, et al. Functional brain imaging during anesthesia in humans: effects of halothane on global and regional cerebral glucose metabolism. Anesthesiology 1999; 90: 701-709

22. Alkire MT, Haier RJ, Barker SJ, Shah NK, Wu JC, Kao YJ. Cerebral metabolism during propofol anesthesia in humans studied with positron emission tomography. Anesthesiology 1995; 82: 393-403 